\title{
Características clínicas de adolescentes de sexo masculino con trastornos de la conducta alimentaria. Estudio de casos clínicos
}

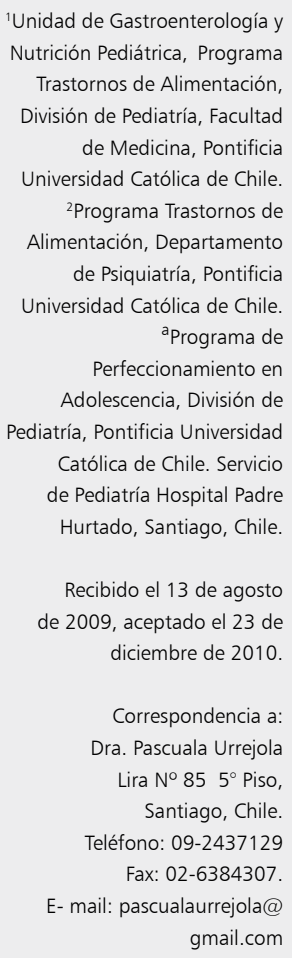

\author{
FRANCISCA SALAS $S^{\text {a }}$, M ISABEL HODGSON ${ }^{1}$, \\ DOLLY FIGUEROA ${ }^{2}$, PASCUALA URREJOLA ${ }^{1}$
}

\section{Clinical features of adolescent males with eating disorders}

Background: There is a higher prevalence of eating disorders (ED) among adolescent females. These are less common in men and there are clinical differences between genders. Aim: To describe the clinical features of adolescent males with ED. Material and Methods: Review of 38 medical records of male patients with ED seen in an ED Unit in Chile, between 2001 and 2009. Results: Mean age at first medical visit was $14.4 \pm 2.2$ years. Symptoms appeared $9.5 \pm 8.9$ months prior to the first visit. Anorexia Nervosa (AN) of the restricting type was diagnosed in 21 patients (55.2\%), with a mean weight loss of $11.2 \mathrm{~kg} \pm 9.4 \mathrm{~kg}$. In $37 \%$ of patients, there was a past medical history of overweight. All patients either increased or maintained weight during follow up. Sixty three percent had a psychiatric co-morbidity at first visit. After 3 months of treatment, $82 \%$ of patients remained in the program. Conclusions: There was a delay in diagnosis of ED in men. The high prevalence of AN found in this group is characteristic of patients recently diagnosed with ED. Overweight could be a precipitating factor. Nearly two thirds of male ED patients had a psychiatric comorbidity. This high prevalence may suggest a susceptibility to develop ED in males.

(Rev Med Chile 2011; 139: 182-188).

Key words: Adolescent, male; Anorexia nervosa; Eating disorders.
L

os trastornos de la conducta alimentaria (TCA) constituyen una patología siquiá-

trica, observada principalmente en la adolescencia, cuya frecuencia ha aumentado considerablemente en las últimas décadas ${ }^{1-5}$. En su conjunto, debido a las complicaciones metabólicas y cardiovasculares que producen, generan morbilidad significativa y una mortalidad que puede llegar a $12 \%{ }^{6}$. A pesar de afectar principalmente a mujeres, se ha observado un incremento sostenido en el número de hombres que la padecen ${ }^{4}$, estimándose actualmente que el sexo masculino representa 1 a $5 \%$ de los casos de anorexia nerviosa (AN) y 5 a $15 \%$ de los casos de bulimia nerviosa
(BN), con prevalencias de hasta $6,5 \%$ entre los 14 y 15 años $^{7-14}$.

Las causas que originan los TCA son multifactoriales e incluyen factores individuales, familiares y sociales, entre los que destacan el sobrepeso previo, estilo de crianza, antecedente familiar de TCA y el actual culto a los cuerpos delgados y perfectos $^{15-20}$.

A diferencia de las mujeres, en los hombres la preocupación está más centrada en ganar masa muscular que en perder masa grasa ${ }^{21-23}$. Cuando esto se asocia a retraso puberal, sobrepeso, $\mathrm{o}$ ambos, aumenta el riesgo de desarrollar un $\mathrm{TCA}^{24,25}$. El sobrepeso ha sido relacionado con $37 \%$ de 
los casos de TCA, especialmente de BN, lo que se traduce en que hasta $25 \%$ de los adolescentes hombres inicien dietas hipocalóricas ${ }^{4,16}$. Otros factores desencadenantes son el bullying, la práctica de deportes que enfatizan la delgadez, y los trastornos de la identidad sexual ${ }^{26-28}$.

En hombres es más probable la asociación de TCA con otros trastornos de conducta y de personalidad, lo que podría complicar aun más el cuadro $^{29}$. Raevuori ${ }^{14}$ estudió varones con AN y los comparó con sus gemelos, observando una asociación familiar de síntomas tales como trastornos afectivos, ansiosos y dismorfofobia.

Dado que los TCA son considerados un trastorno propio de las mujeres, muchos hombres que los padecen se avergüenzan de ello, lo que disminuye la consulta médica espontánea, agravando la sintomatología y aumentando la probabilidad de comorbilidad siquiátrica ${ }^{14,30}$.

En la literatura existen pocos trabajos que proporcionen información sobre los factores predisponentes y la evolución de los TCA en hombres. Conocer esta situación podría ayudar a mejorar el diagnóstico y manejo médico en ellos, favoreciendo una detección temprana y su recuperación en forma integral ${ }^{4}$.

El propósito del presente trabajo es describir el comportamiento clínico de un grupo de adolescentes de sexo masculino, consultantes a un programa de trastornos de alimentación (TAL), y describir los aspectos clínicos más relevantes al momento diagnóstico y en su evolución a corto plazo.

\section{Pacientes y Método}

Se realizó un estudio descriptivo retrospectivo de pacientes de sexo masculino con diagnóstico de TCA según los criterios del DSM-IV-TR ${ }^{31}$ (excluyendo el criterio de amenorrea para AN), que consultaron al equipo de TAL del Departamento de Pediatría de la Pontificia Universidad Católica de Chile entre los años 2001 y 2009. Este equipo atiende a pacientes hasta los 20 años y entrega una atención multidisciplinaria a cargo de pediatras nutriólogos, psicólogos y psiquiatras.

El DSM-IV-TR clasifica los TCA en anorexia nervosa (AN) tipo restrictivo, AN tipo compulsivo-purgativo, bulimia nervosa (BN) tipo purgativo (BP), BN tipo no purgativo (BNP) y trastornos de la conducta alimentaria no especificados (TANE). Se usó esta clasificación para categorizar la muestra.

Se revisaron las fichas de los pacientes atendidos por TCA y se analizaron las que correspondían a pacientes de sexo masculino. De cada ficha se extrajeron los siguientes datos: edad, tiempo de evolución, baja de peso, presencia de vómitos, actividad física extracurricular, antecedente de obesidad, uso de medicamentos y comorbilidad siquiátrica. Se excluyeron los pacientes con patología siquiátrica de base, tales como esquizofrenia y psicosis.

En todos los casos, el peso y la talla fueron determinados en ropa interior, en balanza de precisión con cartabón incluido, marca SECA.

Se calculó el índice de masa corporal (IMC) y el $z$ score de IMC ((IMC real-IMC mediana)/1 DS) usando las tablas del CDC-NCHS $2000^{32}$, con los datos obtenidos al ingreso del paciente y a los 3 meses de tratamiento.

\section{Análisis Estadístico}

Se realizó un análisis descriptivo a través de frecuencias absolutas y relativas para las variables categóricas, y estadísticas de tendencia central para las variables numéricas. Para establecer la comparación del IMC ( $z$ score) se verificó la distribución del IMC ( $z$ score) en los distintos subtipos de TCA y en su evolución. Dado que en estos valores no se observó distribución normal se utilizó el test no paramétrico de Friedman. Se consideró diferencia estadísticamente significativa valores de $\mathrm{p}<0,05$. Todos los análisis se realizaron en SPSS versión 16.0 (Chicago, Illinois).

\section{Resultados}

De 600 pacientes con diagnóstico de TCA atendidos entre 2001 y 2009 por el equipo de TAL, $38(6,3 \%)$ fueron hombres.

La edad de la primera consulta fluctuó entre los 9 y 18 años, con un promedio de 14,4 $\pm 2,2$ años (Tabla 1). No se observaron diferencias significativas entre los distintos tipos de TCA.

El tiempo de evolución previo al diagnóstico fue registrado con precisión en $82 \%$ de los pacientes y fluctuó entre 1 y 48 meses, con un promedio de $9,5 \pm 8,9$ meses.

La Tabla 1 muestra la distribución diagnóstica 
Tabla 1. Distribución de frecuencias de edad al consultar según tipo de trastorno alimentario al ingreso

\begin{tabular}{|lcccccc|}
\hline EPC & AR & BP & BNP & ACP & TANE & Total (\%) \\
9-12 años & 3 & 1 & 0 & 0 & 2 & $6(15,7)$ \\
$12,1-14$ años & 4 & 1 & 0 & 0 & 1 & $6(15,7)$ \\
$14,1-16$ años & 9 & 3 & 1 & 2 & 0 & $15(39,4)$ \\
$16,1-18$ años & 5 & 4 & 1 & 0 & 1 & $11(28,9)$ \\
Total (\%) & $21(55,2)$ & $9(23,6)$ & $2(5,2)$ & $2(5,2)$ & $4(10,5)$ & $38(100)$ \\
\hline
\end{tabular}

EPC: edad primera consulta (años, meses). AR: anorexia restrictiva. BP: Bulimia purgativa. BNP: Bulimia no purgativa. ACP: anorexia compulsiva/purgativa. TANE: Trastorno alimentario no especificado.

Tabla 2. Edad al momento del diagnóstico y comparación del IMC en puntaje $z$ al inicio y a los tres meses según tipo de trastorno alimentario

\begin{tabular}{|cccccc|}
\hline $\begin{array}{c}\text { Tipo de trastorno } \\
\text { alimentario }\end{array}$ & Edad [promedio \pm DS $]$ & $\begin{array}{c}\text { zIMC inicio } \\
\text { [promedio } \pm \text { DS }]\end{array}$ & $\begin{array}{c}\text { (N) } \\
\text { zlMC } 3 \text { meses } \\
\text { [promedio } \pm \mathbf{D S} \text { ] }\end{array}$ & (N) \\
AR & $14,5 \pm 2,3$ & $-0,39 \pm 0,59$ & $(21)$ & $-0,29 \pm 0,54$ & $(17)$ \\
BP & $15,5 \pm 2,2$ & $-0,24 \pm 0,47$ & $(9)$ & $-0,03 \pm 0,45$ & $(8)$ \\
TANE & $12,5 \pm 3,3$ & $-1,09 \pm 0,33$ & $(4)$ & $-0,28 \pm 1,16$ & $(3)$ \\
ACP & $15,5 \pm 0,7$ & $-0,51 \pm 0,32$ & $(2)$ & $-0,61 \pm 0$ & $(1)$ \\
BNP & $15,5 \pm 2,1$ & $-0,16 \pm 0,53$ & $(2)$ & $-0,19 \pm 0,51$ & $(2)$ \\
\hline
\end{tabular}

AR: Anorexia restrictiva. BP: Bulimia purgativa. TANE: Trastorno alimentario no especificado. ACP: Anorexia compulsiva/purgativa. BNP: Bulimia no purgativa. DS: desviación estándar. N: número de pacientes.

al ingreso. Se diagnosticó AN tipo restrictivo en $55,2 \%$ de los pacientes $(\mathrm{n}=21)$, seguido por 9 casos $(23,6 \%)$ de BN tipo purgativo. La edad de presentación de AN tipo restrictivo y $\mathrm{BN}$ tipo purgativo se concentró entre los 14 y 18 años en 26 casos $(68,3 \%)$.

La baja de peso previa al diagnóstico, fue consignada en 79\% de los casos. En los pacientes con AN fue de 11,2 $\pm 9,4 \mathrm{~kg}$, con un rango de $0 \mathrm{a}$ $20 \mathrm{~kg}$. El paciente que no perdió peso tenía 11,6 años, diagnóstico de AN tipo restrictivo y una detención en el incremento de peso esperado para su edad. En los pacientes con BN, la baja de peso fluctuó entre 0 y $42 \mathrm{~kg}$. El paciente que perdió 42 $\mathrm{kg}$ fue diagnosticado como BN tipo purgativo a los 16 años. Dentro de sus antecedentes destaca la presencia de rasgos de personalidad obsesivos (sin constituir trastorno) y una organización limítrofe de personalidad. Del total de pacientes con BN, tres notificaron no haber perdido peso, dos de ellos tenían BN tipo no purgativo y el tercero una $\mathrm{BN}$ tipo purgativo. Estos tres pacientes comparten una dismorfofobia severa y 12 meses de evolución previa al diagnóstico.

En la Tabla 2 se compara el IMC, en puntaje $\mathrm{z}$, al inicio y a los 3 meses de tratamiento en los diferentes grupos diagnósticos. De los 38 pacientes iniciales, sólo 31 (82\%) continuaron en control a los 3 meses. El mayor compromiso de IMC se presentó en los TANE, coincidiendo con el promedio de edad más bajo. En la evolución se observó una tendencia (no significativa) a la mejoría en los casos de AN tipo restrictivo, BN tipo purgativo y TANE. Hubo mantención de la situación nutricional en AN tipo compulsivo-purgativo y BN tipo purgativo.

En la Tabla 3 se muestra la relación entre sobrepeso u obesidad y tipo de TCA. El 37\% de los pacientes tuvo antecedente de sobrepeso al ingreso. En los pacientes con BN tipo purgativo la asociación se elevó a 78\%.

El 57,8\% de los pacientes tuvo antecedentes de realizar actividad física excesiva.

Del total de los pacientes, 7 fueron derivados 
Tabla 3. Tabla de contingencia entre antecedentes de sobrepeso u obesidad según tipo de trastorno alimentario

\begin{tabular}{|c|c|c|c|c|c|c|c|c|c|c|c|c|}
\hline \multirow[t]{2}{*}{ ASPO } & \multicolumn{2}{|c|}{ AR } & \multicolumn{2}{|c|}{ BP } & \multicolumn{2}{|c|}{ TANE } & \multicolumn{2}{|c|}{ ACP } & \multicolumn{2}{|c|}{ BNP } & \multicolumn{2}{|c|}{ Total } \\
\hline & $\mathbf{n}$ & $(\%)$ & $\mathbf{n}$ & (\%) & $\mathbf{n}$ & (\%) & $\mathbf{n}$ & $(\%)$ & $\mathbf{n}$ & $(\%)$ & n & (\%) \\
\hline Sí & 7 & $(33,3)$ & 7 & $(77,7)$ & 0 & & 0 & & 0 & & 14 & $(36,8)$ \\
\hline No & 14 & $(66,6)$ & 2 & $(22,2)$ & 4 & (100) & 2 & (100) & 2 & (100) & 24 & $(64,2)$ \\
\hline Total & 21 & (100) & 9 & (100) & 4 & (100) & 2 & (100) & 2 & (100) & 38 & (100) \\
\hline
\end{tabular}

ASPO: antecedentes de sobrepeso y/o obesidad. AR: Anorexia restrictiva. BP: Bulimia purgativa. TANE: Trastorno alimentario no especificado. ACP: Anorexia compulsiva/purgativa. BNP: Bulimia no purgativa.

por otro especialista; 5 de ellos por endocrinólogo y 2 por gastroenterólogo. El resto consultó espontáneamente al equipo de TAL.

Todos los pacientes iniciaron tratamiento multidisciplinario con apoyo de psiquiatra y terapeuta familiar. El $63,1 \%$ (24) presentó comorbilidad siquiátrica al momento del diagnóstico. El diagnóstico más frecuente fue el trastorno de ánimo en 10 pacientes; 3 con AN tipo restrictivo y 7 con BN tipo purgativo. Un paciente con TANE presentó un trastorno de ansiedad no especificado. Ocho pacientes presentaron rasgos de personalidad de tipo obsesivo sin llegar a constituir un trastorno (3 AN tipo restrictivo, $1 \mathrm{AN}$ tipo compulsivo-purgativo, 3 BN tipo purgativo y 1 BN tipo no purgativo).

El tratamiento fue iniciado sin indicación de psicofármacos en 55,3\% de los pacientes. De los 17 pacientes que fueron tratados con medicamentos desde el inicio, 13 lo hicieron con inhibidores de la recaptura de serotonina, 4 con benzodiacepinas y 6 con antisicóticos. En ocho casos el tratamiento fue combinado, usando terapia bi o tri asociada.

Un paciente de 15 años, con AN tipo restrictivo, requirió hospitalización por compromiso nutricional importante debido a baja de peso de $15 \mathrm{~kg}$ en un mes.

\section{Discusión}

Son escasos los datos epidemiológicos sobre TCA en hombres ya que la mayoría de los estudios los excluyen. Esto ocurre por ser menos frecuentes que en mujeres, y porque la amenorrea es uno de los criterios diagnósticos. Además, todavía son considerados una patología femenina, lo que implica que los profesionales de la salud y los padres, no estén sensibilizados, dificultando su detección y derivación. También ocurre que muchos adolescentes varones temen sentirse estigmatizados, por lo que no buscan ayuda ${ }^{28}$.

El grupo evaluado es la serie más grande reportada en Chile, pese a lo cual el número de casos aún es reducido. En nuestra experiencia, el porcentaje de hombres consultantes por TCA (6,3\% del total en 9 años), es menor a la prevalencia encontrada en países desarrollados ${ }^{10}$. Es probable que en Chile el número de hombres afectados sea mayor, considerando que nuestra población es sesgada, y corresponde sólo a una parte del universo posible de pacientes.

Se desconoce la razón última por la cual los TCA son menos frecuentes en hombres, a pesar de encontrarse casos aislados descritos desde el siglo XVII ${ }^{13}$. Behar et a ${ }^{133}$ proponen que el rol de género femenino, adquirido por el aprendizaje de las normas socioculturales ligadas a la cultura occidental, sería un contribuyente importante en esta diferencia. Otros autores han observado que los factores predisponentes y las manifestaciones serían similares en ambos sexos, previendo en el futuro un aumento sostenido de esta patología en hombres ${ }^{10,20}$.

En nuestra serie, los adolescentes consultantes son de un amplio rango de edad, debido a que el equipo de TAL atiende a niños y adolescentes. La mayor frecuencia de casos se presentó en la adolescencia media y tardía (14 a 18 años) tal como lo han observado otros autores ${ }^{25,35}$. Se ha reportado inicio en edades aun más tardías (18 a 24 años), siendo una de las explicaciones para esto que los cambios puberales en hombres se inician con posterioridad a lo observado en mujeres, $y$ al hecho que algunas de las series más grandes incorporan a hombres de todas las edades, lo que aumentaría el promedio de edad de presentación ${ }^{8,13,17}$. 
En nuestro estudio, el tiempo de evolución previo al diagnóstico fue de 9 meses. Este tiempo de demora entre los primeros síntomas y el diagnóstico es menor al observado en una publicación previa realizada por el mismo equipo ${ }^{34}$, que incluía pacientes en su mayoría mujeres y con un rango más amplio de edad. El menor tiempo de evolución observado, podría explicarse por la mayor severidad de los síntomas observados en los hombres con respecto a las mujeres. Estos datos son importantes de precisar ya que tanto la precocidad en consultar, como la presentación en la adolescencia temprana, son reconocidos índices de buen pronóstico ${ }^{3,4,13,35}$.

En nuestra población, se encontró un porcentaje de AN $(55,2 \%)$ mayor a los trastornos purgativos, lo que difiere con estudios realizados en pacientes con TCA de todas las edades y de ambos sexos, donde el número de casos de hombres con $\mathrm{AN}$ es menor que BN y TANE ${ }^{35}$. Este hallazgo podría deberse a la edad de los pacientes, los que al ser menores podrían compararse con la evolución de los TCA en mujeres al inicio del trastorno.

$\mathrm{Al}$ revisar la evolución de los casos a los 3 meses de tratamiento, se consideró el IMC en puntaje z para evaluar el estado nutricional. Se observó que quienes tendieron a tener mayor compromiso del IMC fueron los pacientes con TANE, todos prepúberes y con comorbilidad siquiátrica. En los pacientes restrictivos, el $z$ score no logra demostrar depleción nutricional a pesar de la importante baja de peso observada, lo que probablemente se explica por la alta prevalencia de malnutrición por exceso. En los pacientes con AN tipo restrictivo, $\mathrm{BN}$ tipo purgativo y TANE hubo una tendencia no significativa a la recuperación del IMC, elemento que no se observó en los pacientes con AN tipo compulsivo-purgativo y $\mathrm{BN}$ tipo no purgativo, sin embargo, el número pequeño de casos en ambos, no permite sacar conclusiones al respecto.

La deserción de pacientes a los 3 meses es un hecho que se observa también en mujeres y en general tiene relación con un rechazo al diagnóstico por parte de los padres y de los propios pacientes.

Al igual que lo observado en series nacionales y extranjeras, $37 \%$ de los pacientes estudiados tuvo el antecedente de sobrepeso u obesidad ${ }^{16,34}$. Ambas condiciones están relacionadas con la aparición de cualquier trastorno de alimentación ${ }^{17}$. El antecedente de malnutrición por exceso es muy relevante en nuestra población, por el incremento vertiginoso de la obesidad en niños y adolescentes, lo cual aumenta el riesgo de TCA en pacientes con otros factores de riesgo.

En hombres, la búsqueda de la perfección corporal está ligada a tener un cuerpo más musculoso $^{21,23}$. Esto lleva a muchos hombres con TCA a realizar ejercicio físico en forma exagerada, y a usar menos dietas, diuréticos y laxantes para controlar el peso, en relación a lo observado en el sexo femenino ${ }^{8}$. En el presente estudio se consignó realización de actividad física excesiva en $58 \%$ de los pacientes, pero al no estar cuantificada su intensidad no es posible sacar mayores conclusiones a este respecto.

Una alta comorbilidad psiquiátrica ha sido descrita en pacientes con TCA, siendo más frecuentes los trastornos afectivos y de personalidad y el consumo de drogas ${ }^{15,19,36}$. En este estudio, $63 \%$ de los pacientes presentaba comorbilidad psiquiátrica al momento del diagnóstico, predominantemente trastornos del ánimo. En los estudios realizados en hombres con TCA, se sugiere que éstos tienen mayor psicopatología del eje I comparados con mujeres ${ }^{12}$. Por otro lado, se ha observado que los trastornos de la identidad sexual son frecuentes, sobre todo en pacientes con $\mathrm{BN}^{27,28}$. Dentro de esta serie no se encontró pacientes que presentaran consumo de drogas ni trastornos de la identidad sexual.

Este estudio es retrospectivo y no tiene un control suficiente de todas las variables. Además, es una muestra pequeña y no disponemos de grupo control, por lo que a pesar de las asociaciones observadas no podemos sacar conclusiones definitivas. Es relevante plantear nuevos estudios prospectivos para definir mejor el perfil sociodemográfico y clínico; estudios de screening en población general y de riesgo, en la búsqueda de factores predisponentes o protectores, y estudios multicéntricos, con el fin de obtener una muestra adecuada para ampliar el conocimiento en el tema y lograr establecer un perfil local.

\section{Referencias}

1. Mehler P. Bulimia Nervosa. N Engl J Med 2003; 349: 875-81.

2. Yager J, Andersen A. Anorexia Nervosa. N Engl J Med 2005; 353: 1481-8.

3. Mackenzie R, Neinstein L. Anorexia Nervosa and Bulimia. En: Neinstein L. Edit, Adolescent Health Care. 
Maryland, USA: Williams and Wilkins; 1996. p. 564-83.

4. Rome ES, Ammerman S, Rosen DS, Keller RJ, Lock J, Mammel KA, et al. Children and Adolescents With Eating Disorders: The State of the Art. Pediatrics 2003; 111: 98-108.

5. Hoek H, Van Hoeken D. Review of the Prevalence and Incidence of Eating Disorders. Int J Eat Disord 2003; 34 : 383-96.

6. Sullivan PF. Mortality in anorexia nervosa. Am J Psychiatry 1995; 152: 1073-4.

7. Kinzl JF, Mangweth B, Traweger CM, Biebl W. Eatingdisordered behavior in males: The impact of adverse childhood experiences. Int J Eat Dis 1997; 22: 131-1.

8. Carlat DJ, Carmargo CA Jr, Herzog DB. Eating Disorders in Males: A Report on 135 Patients. Am J Psychiatry 1997; 154: 1127-32.

9. Carlat DJ, Camargo CA Jr. Review of bulimia nervosa in males. Am J Psychiatry 1991; 148: 831-43.

10. Kjelsas E, Bjornstrom C, Gotestam G. Prevalence of eating disorders in female and male adolescent (14-15 years). Eating Behaviors 2004; 5: 13-25.

11. Barry A, Lipmann B. Anorexia nervosa in males. Postgrad Med 1990; 87: 161-5.

12. Olivardia R, Pope HG Jr Mangweth B, Hudson JI. Eating disorders in college men. Am J Psy 1995; 152: 1279-85.

13. Fichter M, Krenn H. Eating Disorders in Males. Handbook of Eating Disorders. John Wiley and Son Ltd, 2003, p. 369-83.

14. Raevuori A, Keski-Rahkonen A, Hoek H, Sihvola E, Rissanen A, Kaprio J. Lifetime Anorexia Nervosa in Young Men in the community. Five cases and their co-twins. Int J Eat Disord 2008; 41: 458-63.

15. Lilenfeld LR, Wonderlich S, Riso LP, Crosby R, Mitchell J. Eating disorders and personality: A methodological and empirical review. Clinical Psychology Review 2006; 26 : 299-320.

16. Nicholls D, Viner R. Eating disorders and weight problems. BMJ 2005; 330: 949-54.

17. Neumark-Sztainer D, Wall M, Guo J. Story M, Haines J, Eisenberg M. Obesity, Disordered Eating, and Eating Disorders in a Longitudinal Study of Adolescents: How Do Dieters Fare 5 Years Later? J Am Diet Asoc 2006; 106: 559-68.

18. Haycraft E, Blissett J. Eating disorders symptoms and parenting styles. Appetite 2010; 54: 221-4.

19. Behar R, Arriagada MI, Casanova D. Trastornos de la conducta alimentaria y trastornos afectivos: Un estudio comparativo. Rev Med Chile 2005; 133: 1407-14.

20. Nguyen-Rodríguez S, Unger J, Spruijt-Metz D. Psychological Determinants of Eating in Adolescence. Eat Disord 2009; 17: 211-24.
21. Crisp AH, Toms DA. Primary Anorexia Nervosa or Weight Phobia in the Male: Report on 13 Cases. Br Med J 1972; 1: 334-8.

22. García-Grau E, Fusté A, Miró A, Saldaña C, Bados A. Coping Style and Vulnerability to Eating Disorders in Adolescent Boys. Eur Eat Disorders Rev 2004; 12: 61-7.

23. Toro J, Castro J, Gila A, Pombo C. Assessment of Sociocultural Influences on the Body Shape Model in Adolescent Male with Anorexia Nervosa. Eur Eat Disorders Rev 2005; 13: 351-9.

24. Fisher M, Fornani V. Gynecomastia as a precipitant of eating disorders in adolescent males. Int J Eat Dis 1990; 9: 115-9.

25. Modan-Moses D, Yaroslavsky A, Novikov I, Segev S, Toledano A, Miterany E, et al. Stunting of Growth as a Major Feature of Anorexia Nervosa in Male Adolescents. Pediatrics 2003; 111: 270-6.

26. Kaltiala-Heno R, Rimpelä M, Rantanen P, Rimpelä A. Bullying at school -an indicator of adolescents at risk for mental disorders. J Adolesc 2000; 23: 661-74.

27. Boroughs M, Thompson JK. Exercise status and sexual orientation as moderators of body image disturbance and eating disorders in males. Int J Eat Dis 2002; 31: 307-11.

28. Herzog DB, Norman DK, Gordon C, Pepose M. Sexual conflict and eating disorders in 27 males. Am J Psychiatry 1984; 141: 989-990.

29. Woodside B, Garfinkel PE, Lin E, Goering P, Kaplan A, Goldbloom DS, et al. Comparisons of Men With Full or Partial Eating Disorders, Men Without Eating Disorders, and Women With Eating Disorders in the Community. Am J Psychiatry 2001; 158: 570-4.

30. Spindler A, Milos G. Links between eating disorder symptom severity and psychiatric comorbidity. Eating Behaviors 2007; 8: 364-73.

31. Lopez-Ibor JJ, Valdez M. DSM-IV-TR: Brevario Criterios diagnósticos. Barcelona, España: Editorial Masson, SA; 2002. p. 249-52.

32. Ogden C, Kuczmarski RJ, Flegal KM, Mei Z, Guo S, Wei $\mathrm{R}$, et al. Centers for Disease Control and Prevention 2000 Growth Charts for the United States: Improvements to the 1977 National Center for Health Statistics Version. Pediatrics 2002; 109: 45-60.

33. Behar R. La identidad de género en la etiopatogenia de los trastornos del hábito del comer. En Behar R, Figueroa G., Editores, Anorexia Nerviosa y Bulimia. Santiago, Chile: Editorial Mediterráneo Ltda.; 2004. p. 55-72.

34. Cordella P, Lizana P, Urrejola P, Figueroa D, Del Río P, Hodgson I. Programa de trastornos de alimentación en adolescentes y jóvenes chilenos: variaciones a 6 meses de tratamiento. Rev Med Chile 2006; 134: 973-80. 
35. Fisher M. Treatment of eating disorders in Children, Adolescents, and Young Adults. Pediatr Rev 2006; 27: 5-16.

36. Halmi KA, Sunday SR, Strober M, Kaplan A, Woodside
DB, Fichter M, et al. Perfectionism in Anorexia Nervosa: Variation by Clinical Subtype, Obsessionality, and $\mathrm{Pa}-$ thological Eating Behavior. Am J Psychiatry 2000; 157: 1799-805. 